IMAGE

\title{
Insights image for "Evidence of increased hypoxia signalling in fetal liver from maternal nutrient restriction in mice"
}

Bethany N. Radford ${ }^{1,2}$ and Victor K. M. Han ${ }^{1,2,3}$

Pediatric Research (2020) 87:611; https://doi.org/10.1038/s41390-019-0555-9

A schematic representation for the proposed mechanism of chronic hypoxia signalling in response fetal undernutrition. Prolonged reduction in delivery of nutrients to the fetus results in increased stability of hypoxia inducible signalling factor (HIFs) alpha subunit protein levels, either through redistribution of fetal circulation and/or nutritional signalling. Late in gestation (E18.5) increased HIF-2a levels induced transcription of genes involved in metabolism, epigenetic regulation and negative regulators of the HIF pathway. These transcriptional pathways are important in proper liver development and growth, and such changes could contribute to reduced liver size. These alterations could regulate fetal survival and impact long term metabolic changes observed in offspring from maternal nutrient restriction.

\section{REFERENCE}

Radford, B. N., Han, V. K. M. Evidence of increased hypoxia signaling in fetal liver from maternal nutrient restriction in mice. Pediatr Res. (2019). https://doi.org/10.1038/ s41390-019-0447-z [Epub ahead of print].

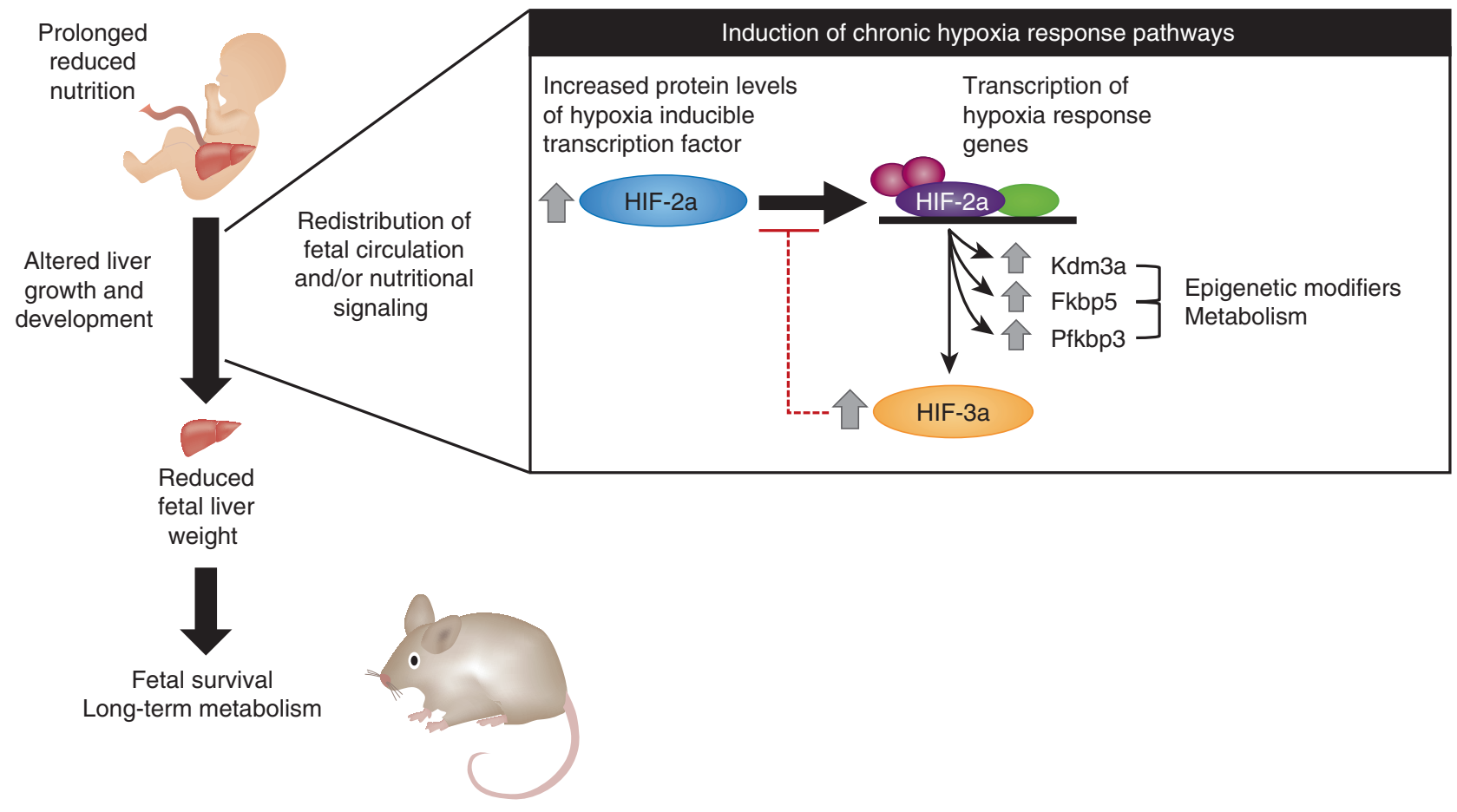

\footnotetext{
'Departments of Biochemistry, Schulich School of Medicine \& Dentistry, Western University, London, ON, Canada; ${ }^{2}$ Children's Health Research Institute, London, ON, Canada and ${ }^{3}$ Departments of Pediatrics, Schulich School of Medicine \& Dentistry, Western University, London, ON, Canada Correspondence: Victor K. M. Han (Victor.Han@lhsc.on.ca)
}

Received: 27 August 2019 Accepted: 27 August 2019

Published online: 4 September 2019 